\title{
Case of Staphylococcus schleiferi Vascular Access Infections with a Hemodialysis Patient
}

\section{Joon Won Chae', Shi Nae Yu', Eun Jung Lee'}

'Division of Infectious Diseases, Department of Internal Medicine, Soonchunhyang University Seoul Hospital, Soonchunhyang University College of Medicine, Seoul; ${ }^{2}$ Division of Infectious Diseases, Department of Internal Medicine, Soonchunhyang University Cheonan Hospital, Soonchunhyang University College of Medicine, Cheonan, Korea

\begin{abstract}
Staphylococcus schleiferi has been infrequently reported as a human pathogen. We experienced the case of vascular access infection by Staphylococcus schleiferi in a 65-year-old hemodialysis patient. Metastatic infection occurred despite the removal of infected focus and appropriate antibiotic therapy. This case highlights the importance of considering Staphylococcus schleiferi as a true pathogen in vascular access infection with hemodialysis patients.
\end{abstract}

Keywords: Staphylococcus schleiferi; Persistent bacteremia; Renal dialysis

\section{INTRODUCTION}

Staphylococcus schleiferi was first described in 1988 by Freney et al. [1], and it has been considered as a member of persistent or transient human pre-axillary skin flora [2]. Although the virulence factors such as deoxyribonuclease, lipase, esterase, protease, beta-hemolysin, and adherence have been described, there were only limited reports implicating it as the causative pathogen of several human infections $[2,3]$. However, distinguishing between infection and contamination presents an important challenge for clinicians. Here, we report the case of vascular access infection caused by S. schleiferi.

\section{CASE REPORT}

A 65-year-old man on hemodialysis was admitted with sensation of heat and pain at the insertion site of the left femoral tunneled cuffed catheter. He previously received kidney transplantation 25 years ago, but is on hemodialysis due to the graft rejection after 4 years. He had been repeatedly operated thereafter for vascular accesses because of recurrent infections. Eight months earli- er, a left axillo-right jugular necklace type arteriovenous bridge graft (AVBG) was performed while using the left femoral tunneled cuffed catheter for hemodialysis. On the day of admission, the initial physical examination revealed redness at the insertion site of the left femoral tunneled cuffed catheter. His temperature was $40^{\circ}$ C, the blood pressure was $160 / 90 \mathrm{~mm} \mathrm{Hg}$, and the heart rate was 110 beats/min. Laboratory findings were leukocyte 23,400 cells $/ \mu \mathrm{L}$ (neutrophil 91\%), hemoglobin $13.3 \mathrm{~g} / \mathrm{dL}$ (normal range, 12-16 g/dL), platelet count 134,000/ $\mu \mathrm{L}$ (normal range, 130,000-450,000/ $\mu \mathrm{L}$ ), C-reactive protein $5.5 \mathrm{mg} / \mathrm{dL}$ (normal range, $0-0.5 \mathrm{mg} / \mathrm{dL}$ ), and procalcitonin $35 \mathrm{ng} / \mathrm{mL}$ (normal range, $0-0.5 \mathrm{ng} / \mathrm{mL}$ ). Methicillin-resistant $S$. schleiferi were isolated by MicroScan WalkAway-96 plus system (Siemens, Munich, Germany) in all three pairs of blood culture. Femoral tunneled cuffed catheter was removed on the day of admission, and S. schleiferi was isolated from the femoral catheter tip culture. Vancomycin was started empirically on the day of admission. There was no vegetation on transesophageal echocardiography and there was no evidence of endophthalmitis on ophthalmologic consultation, no evidence of hematogenous vertebral osteomyelitis on computerized tomography 7 days after admission. There was no evidence of pneumonia on 
chest X-ray. After 14 days of vancomycin therapy, blood cultures remained positive despite the fact that adequate vancomycin trough levels were maintained $(15-23.9 \mu \mathrm{g} / \mathrm{mL})$. Fifteen days after admission, a sensation of heat and pain were experienced at the left axillo-right jugular AVBG site. We therefore considered it as a sign of metastatic infection and infected graft, and bypass graft excision was done on that day. S. schleiferi was isolated on graft material and pus obtained during surgery. The blood culture became negative 6 days after surgery and he was maintained on vancomycin $(5 \mathrm{mg} / \mathrm{kg}$ ) for 2 weeks following resolution of bacteremia. A follow-up 6 months after discharge revealed that the infection has not recurred.

\section{DISCUSSION}

Infections are the second leading cause of mortality (11.9\%) among hemodialysis patients [4], and arteriovenous vascular graft is more commonly associated. In a retrospective study on vascular access infections in 224 hemodialysis patients in Korea, $79.7 \%$ had an arteriovenous vascular graft and $12.5 \%$ had a tunneled cuffed catheter. The most common causative organism was Staphylococcus aureus (62.5\%; methicillin-susceptible, 35.2\%; methicillin-resistant, 27.3\%) [5]. To our knowledge, this is the first reported case of $S$. schleiferi causing vascular access infection in hemodialysis patients and was successfully treated with complete foci removal.

S. schleiferi has two distinct subspecies, which are known as $S$. schleiferi subsp schleiferi and S. schleiferi subsp coagulans. They can be distinguished by activity of tube coagulase and urease. $S$. schleiferi subsp schleiferi is tube coagulase and urease negative, whereas S. schleiferi subsp coagulans is tube coagulase and urease positive [6].

S. schleiferi has shown diverse human involvement including infection of pacemaker wires, endocarditis, extradural abscesses, and meningitis [6,7]. A retrospective study on 28 patients with $S$. schleiferi infection found that this pathogen was most commonly involved with surgical wound infections. The majority of patients in this study were older men (mean age of 64 years), and the most common underlying co-morbidity was malignancy. Infection was nosocomial in 22 cases (78.6\%). Two patients became infected after aorto-femoral bypass grafting and femoro-popliteal bypass grafting for peripheral vascular disease [8].

S. schleiferi subsp coagulans infection in humans is a rare condition. Leung et al. [6] described a case with endocarditis, and
Thibodeau et al. [9] reported a patient with a left ventricular assistance device infection awaiting heart transplantation.

The actual occurrence of S. schleiferi infections is underreported due to the erroneous identification of S. schleiferi as S. aureus in routine laboratory testing. Both strains exhibit beta-hemolysis and are morphologically similar on blood agar. Moreover, S. schleiferi subsp. schleiferi, like S. aureus, produces both a clumping factor and thermonuclease [10]. In one study of S. schleiferi infection, due to previous use of MicroScan identification, 20 cases of $S$. schleiferi infection might have been misdiagnosed, because their corresponding isolates were previously identified as S. aureus [8].

In our case, the tunneled cuffed catheter and AVBG probably acted as a pathogenic factor. In this regard, it is tempting to speculate that specific adhesins and slime produced by this organism could have favored its growth as a biofilm adherent to the graft, starting and maintaining the $S$. schleiferi infection. On the other hand, growth as a biofilm could have protected the staphylococci from antibiotics.

Although we performed an appropriate removal of the tunneled cuffed catheter in the femoral vein and administered appropriate antibiotic therapy in our patient, persistent bacteremia caused metastatic infection of the prepared left axillo-right jugular necklace type AVBG. It was not until the prepared AVBG was removed that $S$. schleiferi bacteremia resolved. Therefore, clinicians should consider vascular access infection in hemodialysis patients in the differential diagnosis of $S$. schleiferi bacteremia and the removal of infected foci is critical for a good prognosis.

\section{REFERENCES}

1. Freney J, Brun Y, Bes M, Meugnier H, Grimont F, Grimont PA, et al. Staphylococcus lugdunensis sp. nov. and Staphylococcus schleiferi sp. nov., two species from human clinical specimens. Int J Syst Evol Microbiol 1988;38:168-72.

2. Ozturkeri H, Kocabeyoglu O, Yergok YZ, Kosan E, Yenen OS, Keskin K. Distribution of coagulase-negative staphylococci, including the newly described species Staphylococcus schleiferi, in nosocomial and community acquired urinary tract infections. Eur J Clin Microbiol Infect Dis 1994;13:1076-9.

3. Hebert GA. Hemolysins and other characteristics that help differentiate and biotype Staphylococcus lugdunensis and Staphylococcus schleiferi. J Clin Microbiol 1990;28:2425-31.

4. Jin DC. Dialysis registries in the world: Korean Dialysis Registry. Kidney Int Suppl (2011) 2015;5:8-11.

5. Park SY, Lee EJ, Kim TH, Jeon MH, Choo EJ. Clinical characteristics and outcome of vascular access infections in hemodialysis patients. Infect Chemother 2011;43:343-8.

6. Leung MJ, Nuttall N, Mazur M, Taddei TL, McComish M, Pearman JW. 
Case of Staphylococcus schleiferi endocarditis and a simple scheme to identify clumping factor-positive staphylococci. J Clin Microbiol 1999; 37:3353-6.

7. Celard M, Vandenesch F, Darbas H, Grando J, Jean-Pierre H, Kirkorian G, et al. Pacemaker infection caused by Staphylococcus schleiferi, a member of the human preaxillary flora: four case reports. Clin Infect Dis 1997; 24:1014-5.

8. Hernandez JL, Calvo J, Sota R, Aguero J, Garcia-Palomo JD, Farinas MC. Clinical and microbiological characteristics of 28 patients with Staphylo- coccus schleiferi infection. Eur J Clin Microbiol Infect Dis 2001;20:153-8. 9. Thibodeau E, Boucher H, Denofrio D, Pham DT, Snydman D. First report of a left ventricular assist device infection caused by Staphylococcus schleiferi subspecies coagulans: a coagulase-positive organism. Diagn Microbiol Infect Dis 2012;74:68-9.

10. Vandenesch F, Lebeau C, Bes M, Lina G, Lina B, Greenland T, et al. Clotting activity in Staphylococcus schleiferi subspecies from human patients. J Clin Microbiol 1994;32:388-92. 